\title{
Adaptive Cross-Layer Distributed Energy-Efficient Resource Allocation Algorithms for Wireless Data Networks
}

\author{
Stefano Buzzi, ${ }^{1}$ H. Vincent Poor, ${ }^{2}$ and Daniela Saturnino ${ }^{1}$ \\ ${ }^{1}$ DAEIMI, University of Cassino, Via G. Di Biasio 43, 03043 Cassino (FR), Italy \\ ${ }^{2}$ School of Engineering and Applied Science, Princeton University, Princeton, NJ 08544, USA \\ Correspondence should be addressed to Stefano Buzzi, buzzi@unicas.it
}

Received 1 February 2008; Revised 18 June 2008; Accepted 31 July 2008

Recommended by Christian Peel

The issue of adaptive and distributed cross-layer resource allocation for energy efficiency in uplink code-division multiple-access (CDMA) wireless data networks is addressed. The resource allocation problems are formulated as noncooperative games wherein each terminal seeks to maximize its own energy efficiency, namely, the number of reliably transmitted information symbols per unit of energy used for transmission. The focus of this paper is on the issue of adaptive and distributed implementation of policies arising from this approach, that is, it is assumed that only readily available measurements, such as the received data, are available at the receiver in order to play the considered games. Both single-cell and multicell networks are considered. Stochastic implementations of noncooperative games for power allocation, spreading code allocation, and choice of the uplink (linear) receiver are thus proposed, and analytical results describing the convergence properties of selected stochastic algorithms are also given. Extensive simulation results show that, in many instances of practical interest, the proposed stochastic algorithms approach with satisfactory accuracy the performance of nonadaptive games, whose implementation requires much more prior information.

Copyright (C) 2009 Stefano Buzzi et al. This is an open access article distributed under the Creative Commons Attribution License, which permits unrestricted use, distribution, and reproduction in any medium, provided the original work is properly cited.

\section{Introduction and Work Motivation}

Cross-layer design $[1,2]$ has proven to be an effective tool for improving the performance of wireless data networks. The intuition behind this approach is that joint optimization across different layers of the network protocol stack leads to better-performing solutions than those obtained when the network layers are separately optimized. Over the past decade, a considerable amount of work has been carried out in this area, and this has contributed to significant advances in the state-of-the-art knowledge on the design principles of wireless networks.

In the context of multiuser data networks, such as those based on the code-division multiple-access (CDMA) air interface, cross-layer design has primarily addressed the integration of physical layer issues such as multiuser detection, error correction, and channel estimation, with higher layers functions such as power control, call admission control, packet collision resolution, and so on. In this framework, cross-layer resource allocation procedures have recently been developed through a game-theoretic approach for maximizing the uplink terminals' energy-efficiency, which is defined here as the number of reliably transmitted bits per unit of energy used for transmission. Indeed, game theory [3], a branch of mathematics that has been applied primarily in economics and other social sciences to study the interactions among several autonomous subjects with contrasting interests, has been proven useful for the design and analysis of communication systems, primarily with application to resource allocation algorithms [4], and in particular, to power control [5]. In [6-8], for a multiple access wireless data network, both non-cooperative and cooperative games are introduced, wherein each user (i.e., terminal) chooses its transmit power in order to maximize its own utility, defined as the ratio of the throughput to transmit power. While the above studies consider the issue of power control assuming that conventional matched filters are used by the receiver, paper [9], adopting a cross-layer design philosophy, considers the problem of joint linear receiver design and power control so as to maximize the utility of each user. It is shown here that the inclusion of receiver design in the considered game brings remarkable advantages, 
and also results based on the powerful large-system analysis are presented. The results of [9] have been then extended in [10] to the case in which the users' spreading codes are included in the tunable parameters for utility maximization. The study [10] shows that significant performance gains can be obtained through the joint optimization of the spreading code, the transmit power, and the receiver filter for each user. Finally, [11] considers the problem of joint power control and linear receiver optimization for energy efficiency maximization in an asynchronous network subject to multipath.

It should be noted that the solutions proposed in the above-noted studies, although providing a general framework for cross-layer resource optimization through a game theoretic approach, describe (and analyze) nonadaptive solutions based on perfect knowledge of a number of parameters such as the spreading codes, the transmit powers, the propagation channels, and the receive filters for all the users, which are assumed to be obtained offline. In particular, this kind of information is needed to compute the optimal receiver for each terminal and to compute the received signalto-interference-plus-noise ratio (SINR) for each user, which is needed in the power control updates.

This paper, instead, addresses the problem of adaptive and distributed implementation of noncooperative games for energy-efficient resource allocation. The term "adaptive" refers here to the consideration of the more practical and challenging situation in which each user tries to maximize its own utility based on the knowledge of its parameters only, that is, assuming total ignorance of the interference background, and based only on readily available measurements such as the received data. We propose adaptive algorithms that are shown, either analytically or via numerical simulations, to approximate with good-to-excellent accuracy the behavior of nonadaptive implementations of several resource allocation algorithms. Indeed, our results show that these algorithms converge to a neighborhood of the Nash equilibrium (NE) point of the corresponding nonadaptive games.

\subsection{Summary of the Results. The contributions of this paper} may be summarized as follows.

(i) For a single-cell system, we propose adaptive implementations of utility-maximizing noncooperative games, assuming that each user knows its own channel only. First, the case in which the transmit power and the linear uplink receiver may be tuned is considered; then an additional optimization with respect to the users' spreading codes is also considered. For the former case, some analytical considerations on the convergence properties of the proposed adaptive algorithms are also given. For the latter case, instead, we give a proof of the existence and uniqueness of the NE using the concept of separable games [12].

(ii) For the case of transmit power control and choice of the uplink linear receiver in a single-cell system, we also consider the practically relevant case in which no channel state information is available to the receiver, thus implying that channel coefficients must be estimated based on training sequences. We thus analyze adaptive implementations of the games, taking into account the fact that the channel is not perfectly known at the receiver due to estimation errors. (iii) The above noncooperative resource allocation games are also extended to a multicell network, where out-of-cell interference is explicitly taken into account. Also in this case, we consider two different noncooperative games, with and without optimization with respect to the spreading code of each user. In particular, we will see that when spreading code optimization is performed in a multicell scenario, an NE is not guaranteed to exist. Nonetheless, running spreading code optimization updates is still beneficial, since it leads to a set of spreading codes having a better performance than that of the original set.

1.2. Outline of the Paper. This paper is organized as follows. The next section contains some background information on game theory and on the considered utility function. Section 3 deals with adaptive implementations of resource allocation algorithms based on noncooperative games, assuming that each user knows its own channel coefficient. The case in which this information is not available at the receiver and channel estimation is to be performed instead is considered in Section 4. While Sections 3 and 4 refer to a singlecell network, Section 5 focuses on a multicell network. Here, adaptive implementations of resource allocation algorithms are discussed and analyzed. In Section 6, numerical results, corroborating the validity of the proposed adaptive algorithms, are presented and discussed. Finally, Section 7 contains concluding remarks.

\section{Game-Theoretic Approach to Energy Efficiency Maximization}

Consider the uplink of a synchronous, direct-sequence codedivision multiple-access (DS/CDMA) network with $K$ users and processing gain $N$, and subject to flat fading.

Assume that each mobile terminal sends its data in packets of $M$ bits, and that it is interested both in having its data received with error probability as small as possible at the access point (AP), and in making careful use of the energy stored in its battery. Obviously, these are conflicting goals, since error-free reception may be achieved by increasing the received signal-to-noise ratio, that is, by increasing the transmit power, which of course comes at the expense of battery life. (Of course there are many other strategies to lower the data error probability, such as the use of error-correcting codes, diversity exploitation, and implementation of optimal reception techniques at the receiver. Here, however, we are mainly interested in energy efficient data transmission and power usage, so we consider only the effects on energy efficiency of varying the transmit power, the receiver, and (possibly) the spreading code. The effects of the other techniques are generally additive to those achieved through the methods described in this paper. A useful approach to quantify these conflicting goals is to define the utility of the $k$ th user as the ratio of its throughput, denoted by $T_{k}$ and defined as the number of information bits that are received with no error in unit time, to its transmit power, denoted by $p_{k}[6,7]$, that is,

$$
u_{k}=\frac{T_{k}}{p_{k}} .
$$




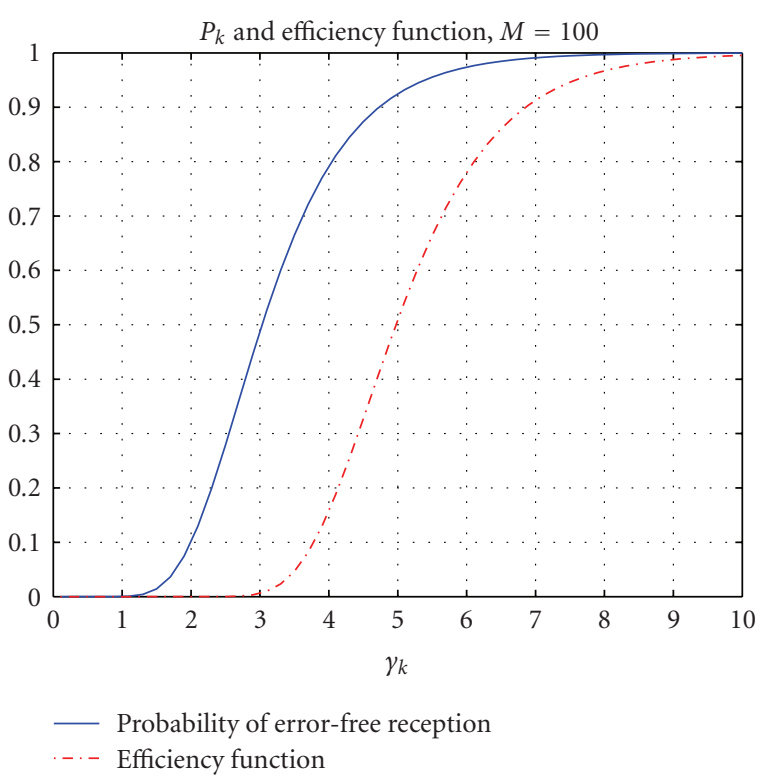

FIGURE 1: Comparison of probability of error-free packet reception and efficiency function versus receive SINR and for packet size $M=$ 100. Note the S-shape of both functions.

Note that $u_{k}$ is measured in bit/Joule, that is, it represents the number of successful bit transmissions that can be made for each Joule of energy used for transmission. Denoting by $R$ the common rate of the network (extension to the case in which each user transmits with its own rate $R_{k}$ is quite straightforward) and assuming that each packet of $M$ symbols contains $L$ information symbols and $M-L$ overhead symbols, reserved, for example, for channel estimation and/or parity checks, the throughput $T_{k}$ can be expressed as

$$
T_{k}=R \frac{L}{M} P_{k}
$$

where $P_{k}$ denotes the probability that a packet from the $k$ th user is received error-free. In the considered DS/CDMA setting, the term $P_{k}$ depends formally on a number of parameters such as the spreading codes of all the users, their channel coefficients, and their transmit powers. However, a customary approach is to model the multiple access interference as a Gaussian random process, and assume that $P_{k}$ is an increasing function of the $k$ th user's SINR $\gamma_{k}$, which is naturally the case in many practical situations. For mathematical reasons, as explained in $[7,9,10]$ and not reported here for the sake of brevity, a customary approach is to replace $P_{k}$ with an efficiency function $f\left(\gamma_{k}\right)$, which is an S-shaped, nondecreasing function, converging to unity for increasing $\gamma_{k}$ and approaching zero for $\gamma_{k} \rightarrow 0$, and with a continuous first-order derivative. A widely accepted efficiency function is

$$
f\left(\gamma_{k}\right)=\left(1-e^{-\gamma_{k}}\right)^{M},
$$

this is indeed a mathematically tractable and widely accepted substitute for the true probability of correct packet reception for the signaling model to be treated in this paper (see Section 3). Although the results of this paper apply to any efficiency function having the above-noted properties, in the following we will adopt the model (3), and we also assume that the efficiency function is the same for all the users. A plot of the efficiency function, and of the corresponding probability of error-free packet reception is represented in Figure 1. Substituting (2) into (1) and replacing the probability $P_{k}$ with the efficiency function (3), we thus obtain the following expression for the $k$ th user's utility:

$$
u_{k}=R \frac{L}{M} \frac{f\left(\gamma_{k}\right)}{p_{k}}, \quad \forall k=1, \ldots, K
$$

Based on the above definitions, we can consider how each user can maximize its own utility and how this maximization will affect other users' utilities. In particular, we are interested in a noncooperative scenario, wherein each user tries to maximize its own utility autonomously and selfishly, that is, with no care for other users' utilities, as happens in a CDMA wireless data network. Game theory provides a useful framework to analyze these scenarios and to study whether a stable point exists. Formally, a noncooperative game $g$ can be described as the triplet $g=\left[\mathcal{K},\left\{\wp_{k}\right\},\left\{u_{k}\right\}\right]$, where $\mathcal{K}=\{1,2, \ldots, K\}$ is the set of active users participating in the game, $u_{k}$ is the $k$ th user's utility (4), and $s_{k}$ is the set of possible actions (strategies) that user $k$ can take. As an example, if each user may tune its transmit power only, then we have $\delta_{k}=\left[0, P_{k, \max }\right]$, with $P_{k \text {,max }}$ the maximum allowed transmit power for the $k$ th user.

An NE [3] for the game $g$ is defined as a profile of strategies such that no user can unilaterally improve its own utility by changing its strategy, assuming that the other users' strategies are fixed, that is, the point $\left(s_{1}, \ldots, s_{K}\right) \in s_{1} \times \cdots \times$ $\delta_{K}$ is an NE point if, for every user $k$, we have

$$
u_{k}\left(s_{1}, \ldots, s_{k}, \ldots, s_{K}\right) \geq u_{k}\left(s_{1}, \ldots, s_{k}^{*}, \ldots, s_{K}\right), \quad \forall s_{k}^{*} \in s_{k} .
$$

A quick reading of this definition might lead one to think that at NE the users' utilities achieve their maximum values. Actually, this is not the case, since the existence of an NE point does not imply that no other $K$-tuple strategy exists that can lead to an improvement of the utilities of some users while not decreasing the utilities of the remaining ones. These latter strategies are usually said to be Paretooptimal [3]. Otherwise stated, if some sort of cooperation were possible, users might agree to simultaneously switch to a different strategy $K$-tuple, so as to improve the utility of some, if not all, active users, while not decreasing the utility of the remaining ones. Since this paper concerns adaptive and distributed implementations of resource allocation algorithms, we are interested here in NE points rather than in Pareto-optimal equilibria. However, it has also been shown that, for the noncooperative games to be considered in the sequel, the NE points are in many cases not really far from the Pareto-frontier $[9,10]$. 


\section{Cross-Layer Resource Allocation in Single-Cell Networks: Known Channel}

In a single-cell network, out-of-cell interference is neglected and the signal received at the AP in the $n$th symbol interval, say $\mathbf{r}(n)$, can be modeled as an $N$-dimensional data vector:

$$
\mathbf{r}(n)=\sum_{k=1}^{K} \sqrt{p_{k}(n)} h_{k} b_{k}(n) \mathbf{s}_{k}(n)+\mathbf{w}(n)
$$

where $p_{k}(n)$ is the transmit power (to simplify subsequent notation, we assume that the transmitted power $p_{k}$ subsumes also the gain of the transmit and receive antennas) of the $k$ th user at epoch $n, b_{k}(n) \in\{-1,1\}$ is the information symbol of the $k$ th user in the $n$th symbol interval, and $h_{k}$ is the real- (we assume here, for simplicity, a real channel model; generalization to practical channels, with $I$ and $Q$ components, is straightforward) channel gain between the $k$ th user's transmitter and the AP; the actual value of $h_{k}$ depends on both the distance of the $k$ th user's terminal from the AP and the channel fading fluctuations. The $N$ dimensional vector $\mathbf{s}_{k}(n)$ is the spreading code of the $k$ th user at epoch $n$; we assume that the entries of $\boldsymbol{s}_{k}(n)$ are real and that $\mathbf{s}_{k}^{T}(n) \mathbf{s}_{k}(n)=\left\|\mathbf{s}_{k}(n)\right\|^{2}=1$, with $(\cdot)^{T}$ denoting the transpose. Finally, $\mathbf{w}(n)$ is the ambient noise vector in the $n$th symbol interval, which we assume to be a zero-mean white Gaussian random process with covariance matrix $\left(\mathcal{N}_{0} / 2\right) \mathbf{I}_{N}$, with $\mathbf{I}_{N}$ the identity matrix of order $N$. An alternative and compact representation of (6) is given by

$$
\mathbf{r}(n)=\mathbf{S}(n) \mathbf{P}^{1 / 2}(n) \mathbf{H b}(n)+\mathbf{w}(n)
$$

where $\mathbf{S}(n)=\left[\mathbf{s}_{1}(n), \ldots, \mathbf{s}_{K}(n)\right]$ is the $N \times K$-dimensional spreading code matrix, $\mathbf{P}(n)$ and $\mathbf{H}$ are $K \times K$ dimensional diagonal matrices, whose diagonals are $\left[p_{1}(n), \ldots, p_{K}(n)\right]$ and $\left[h_{1}, \ldots, h_{K}\right]$, respectively, and finally $\mathbf{b}(n)=\left[b_{1}(n), \ldots, b_{K}(n)\right]^{T}$ is the $K$-dimensional vector of the data symbols. Assuming that a linear receiver is used to detect the data symbol $b_{k}(n)$, it is easily seen that the SINR $\gamma_{k}(n)$ can be written as

$$
\gamma_{k}(n)=\frac{p_{k}(n) h_{k}^{2}\left(\mathbf{d}_{k}^{T}(n) \mathbf{s}_{k}(n)\right)^{2}}{\left(\mathcal{N}_{0} / 2\right)\left\|\mathbf{d}_{k}(n)\right\|^{2}+\sum_{i \neq k} p_{i}(n) h_{i}^{2}\left(\mathbf{d}_{k}^{T}(n) \mathbf{s}_{i}(n)\right)^{2}},
$$

where $\mathbf{d}_{k}(n)$ is the $N$-dimensional vector representing the receive filter for the user $k$ at epoch $n$.

3.1. Power Control and Choice of the Linear Receiver. Let us now consider the situation in which each user is allowed to tune its transmit power and its linear uplink receiver in order to maximize its own utility, that is, the strategy set is $\wp_{k}=$ $\left[0, P_{k, \max }\right] \times \mathcal{R}^{N}$, with $\times$ denoting the Cartesian product and $\mathcal{R}^{N}$, with $\mathcal{R}$ the real line, defines the set of all possible linear receive filters. The game is thus equivalent to the following maximization problem:

$$
\max _{s_{k}} u_{k}=\max _{p_{k}, \mathbf{d}_{k}} u_{k}\left(p_{k}, \mathbf{d}_{k}\right), \quad \forall k=1, \ldots, K,
$$

and given (4), it can be written as

$$
\max _{p_{k}, \mathbf{d}_{k}} \frac{f\left(\gamma_{k}\left(p_{k}, \mathbf{d}_{k}\right)\right)}{p_{k}}, \quad \forall k=1, \ldots, K
$$

In [9], the following result is given (uniqueness of the linear receiver is meant here and in the following up to an irrelevant positive scaling factor).

Proposition 1. The noncooperative game defined in (9) admits a unique NE point $\left(p_{k}^{*}, \mathbf{d}_{k}^{*}\right)$, where $\mathbf{d}_{k}^{*}$ is the vector of minimum mean square error (MMSE) receiver coefficients, and $p_{k}^{*}=\min \left\{\bar{p}_{k}, P_{k, \max }\right\}$, with $\bar{p}_{k}$ the kth user's transmit power such that $\gamma_{k}=\bar{\gamma}$, and with $\bar{\gamma}$ the unique solution of the equation $f(\gamma)=\gamma(d f(\gamma) / d \gamma)$.

In practice, at the NE, each user adopts an MMSE receiver at the AP and its transmit power is such that each user achieves the target SINR $\bar{\gamma}$. If the required power to achieve $\bar{\gamma}$ exceeds the maximum allowed transmit power for some users, then these users transmit at the maximum power and experience an SINR lower than $\bar{\gamma}$. It is also worth noting that, as discussed in [9], the NE point is not far from the Pareto-frontier subject to the equal SINR constraint for all the users. It is easy to see that implementation of this game requires knowledge of a number of parameters of the interfering signals, such as their transmit powers and channel coefficients. Indeed, the $k$ th user's MMSE receiver at epoch $n$ is written as

$$
\mathbf{d}_{k}^{*}(n)=c_{k}(n) \mathbf{R}(n)^{-1} \mathbf{s}_{k}(n)
$$

with $\mathbf{R}(n)$ the covariance matrix of $\mathbf{r}(n)$, and $c_{k}(n)$ a positive constant. Likewise, the transmit power $p_{k}^{*}$ can be set based on the iterative procedure outlined in [5], which indeed requires knowledge of the so-called interference function. To circumvent these difficulties, in what follows we propose an adaptive implementation of the games that is based on readily available measurements such as the received data. The proposed adaptive algorithm is thus amenable to a distributed implementation. For the moment, the distributed algorithm relies on perfect knowledge of the channel coefficient for the user of interest; this hypothesis will be relaxed in the next section.

With regard to the problem of MMSE receiver implementation, we can make use of an adaptive algorithm such as the recursive least squares (RLS) algorithm [13]. The stochastic joint power control and receiver optimization algorithm can be thus implemented as follows. First, we solve the equation $f(\gamma)=\gamma f^{\prime}(\gamma)$, in order to obtain the target SINR $\bar{\gamma}$. Then, for fixed powers, we update the receive filter using, for example, the decision-directed RLS algorithm. Letting $\hat{\mathbf{R}}(0)=\epsilon \mathbf{I}_{N}$, with $\epsilon$ a small positive constant, letting $\lambda$, the forgetting factor, be a close-to-one coefficient, and assuming that the receiver has knowledge of the information symbols $b_{k}(1), \ldots, b_{k}(T)$ (here, we assume the presence of a training 
phase), the following iterations can be considered for each user:

$$
\begin{aligned}
\mathbf{k}(n) & =\frac{\hat{\mathbf{R}}^{-1}(n-1) \mathbf{r}(n)}{\lambda+\mathbf{r}^{T}(n) \widehat{\mathbf{R}}^{-1}(n-1) \mathbf{r}(n)}, \\
\hat{\mathbf{R}}^{-1}(n) & =\frac{1}{\lambda}\left[\hat{\mathbf{R}}^{-1}(n-1)-\mathbf{k}(n) \mathbf{r}^{T}(n) \hat{\mathbf{R}}^{-1}(n-1)\right], \\
e_{k}(n) & =\mathbf{d}_{k}^{T}(n-1) \mathbf{r}(n)-b_{k}(n), \\
\mathbf{d}_{k}(n) & =\mathbf{d}_{k}(n-1)-e_{k}(n) \mathbf{k}(n) .
\end{aligned}
$$

The last equation in (12) represents the update equation for the linear receiver $\mathbf{d}_{k}(\cdot)$. Note that this equation, in turn, depends on the error vector $e_{k}(n)$, which, for $n \leq T$, can be built based on the knowledge of the training symbols $b_{k}(1), \ldots, b_{k}(T)$. Once the training phase is over, real data detection takes place and the error in the third line of (12) is computed according to the equation

$$
e_{k}(n)=\mathbf{d}_{k}^{T}(n-1) \mathbf{r}(n)-\operatorname{sgn}\left[\mathbf{d}_{k}^{T}(n-1) \mathbf{r}(n)\right],
$$

that is, the receiver switches to decision-directed operation. As an alternative to the RLS update in (12), an adaptive and blind least mean squares (LMS) strategy can be used [14], that is,

$$
\mathbf{d}_{k}(n+1)=\mathbf{d}_{k}(n)-\beta(n) \mathbf{d}_{k}^{T}(n) \mathbf{r}(n)\left[\mathbf{r}(n)-\mathbf{s}_{k}^{T}(n) \mathbf{r}(n) \mathbf{s}_{k}(n)\right],
$$

with $\beta(n)$ the step-size for the $n$th iteration. At the beginning, $\mathbf{d}_{k}$ is an arbitrary vector fulfilling the constraint $\mathbf{d}_{k}^{T}(0) \mathbf{s}_{k}(0)=$ 1. Note that, due to computer finite precision effects, the updated vector may not always fulfill this constraint, thus implying that $\mathbf{d}_{k}(n+1)$ must be replaced with its normalized projection onto $\mathbf{s}_{k}(n+1)$, that is,

$$
\mathbf{d}_{k}(n+1)=\mathbf{d}_{k}(n+1)-\left[\mathbf{d}_{k}^{T}(n+1) \mathbf{s}_{k}(n+1)-1\right] \mathbf{s}_{k}(n+1) .
$$

Moreover, with regard to the update of the transmit power, the following LMS iterations can be used (note that the power vector and the receive filter may be updated in parallel):

$$
p_{k}(n+1)=\min \left\{(1-\rho) p_{k}(n)+\rho \ell_{k}(n), P_{k, \max }\right\} .
$$

In the above equations, the step-size $\rho$ is a close-to-zero positive constant and $\ell_{k}(n)$ is a stochastic approximation of the $k$ th entry of the so-called interference vector $\mathbf{I}(\mathbf{p})$ (see [5] for further details), expressed as

$$
\begin{aligned}
& \ell_{k}(n) \\
& =\frac{\bar{\gamma}}{h_{k}^{2}\left(\mathbf{d}_{k}^{T}(n) \mathbf{s}_{k}(n)\right)^{2}}\left[\left(\mathbf{d}_{k}^{T}(n) \mathbf{r}(n)\right)^{2}-p_{k}(n) h_{k}^{2}\left(\mathbf{d}_{k}^{T}(n) \mathbf{s}_{k}(n)\right)^{2}\right] .
\end{aligned}
$$

Note that the update equations in (16) do not require any knowledge of the interference, and so they are amenable to a distributed and decentralized implementation. Summing up, the proposed adaptive algorithms for selfish energyefficiency maximization with respect to the transmit power and linear receiver can be summarized as follows.
(1) Set $\hat{\mathbf{R}}(0)=\epsilon \mathbf{I}_{N}, \mathbf{d}_{k}(0)=\mathbf{s}_{k}$ and $p_{k}(0)=P_{k, \max } / 2$, $\forall k=1, \ldots, K$. Set $n=0$.

(2) Set $n=n+1$.

(3) Update $\mathbf{d}_{k}(n)$ using either the RLS iterations (12) or the blind LMS iteration (14), for $k=1, \ldots, K$.

(4) Compute $\ell_{k}(n)$ using (17) for any $k=1, \ldots, K$, and send it back to the user terminals.

(5) The user terminals can now update their transmit powers based on (16).

(6) Go to step 2.

Note that, in each symbol interval, the receive filter and the transmit power are jointly updated by all the active users.

3.2. Convergence Results. A convergence analysis of the algorithm presented at the end of the previous section is quite difficult. Here we present a convergence analysis for a slightly modified version of the above illustrated adaptive implementation of the game, assuming that a large number of blind LMS receiver updates (14) are performed in between consecutive power updates.

According to [14], if the step-size sequence in (14) fulfills the conditions

$$
\sum_{m=0}^{\infty} \beta(m)=\infty, \quad \sum_{m=0}^{\infty} \beta^{2}(m)<\infty,
$$

then assuming that the power vector $\mathbf{p}(\cdot)$ remains constant, (14) converges almost surely to $\mathbf{d}_{k}^{*}(n)$ of $(11)$ as $m \rightarrow \infty$. Moreover, iteration (14) also converges to $\mathbf{d}_{k}^{*}(n)$ in the mean square sense as long as the step-size sequence fulfills the first condition in (18) and, for increasing $m, \beta(m) \rightarrow 0$ [15]. As a consequence, if sufficiently many iterations (14) are performed between consecutive power updates, the estimated receiver filter $\mathbf{d}_{k}(n)$ tends to be coincident with the MMSE receiver $\mathbf{d}_{k}^{*}(n)$ of (11). In practice, however, we may not be able to perform enough iterations between two consecutive power updates to achieve convergence, so it is reasonable to assume that, at the generic $n$th iteration, the output of the blind LMS iteration can be expressed as

$$
\mathbf{d}_{k}(n)=\mathbf{d}_{k}^{*}(n)+\tilde{\mathbf{w}}_{k}(n)
$$

where $\tilde{\mathbf{w}}_{k}(n)$ is an estimation error. Since (14) converges to $\mathbf{d}_{k}^{*}(n)$ also in the mean square sense, it is always possible [15] to choose the step-size and the number of iterations such that the condition

$$
\mathrm{E}\left[\left\|\tilde{\mathbf{w}}_{k}(n)\right\|^{2} \mid \mathbf{p}(n)\right] \leq \varepsilon(n) K_{0},
$$

holds, where $K_{0}$ is an arbitrary positive constant and $\varepsilon(n)$ is a sequence such that $1 \geq \varepsilon(n) \geq 0$. Under these conditions, it can be shown easily that the stochastic interference function (17) is a quasistandard function, in the sense specified in [15], thus implying that the LMS-based power control update (16) converges almost surely to the power vector resulting from the nonadaptive power control iterations. 
Summing up, the above considerations prove the convergence of the algorithm at the end of Section 3.1 with blind LMS receiver updates under the assumption that a large number of receiver update iterations are performed between two consecutive power control updates. Otherwise stated, we have here considered the case in which step 3 in the recipe at the end of Section 3.1 is replaced by the following step.

(3) Update $\mathbf{d}_{k}(n)$ using the blind LMS iteration (14), for $k=1, \ldots, K$; if $(20)$ is not fulfilled for any $k=1, \ldots, K-1$, go to step 2 .

Although our analysis can be applied to such modified algorithm, numerical results have shown that the adaptive algorithm at the end of Section 3.1 has excellent convergence behavior. Unfortunately, in this case the estimation errors in the receiver and power iterations become correlated, and this is the source of difficulty in providing analytical convergence results.

\subsection{Power Control, Spreading Code Allocation, and Choice of} Linear Receiver. Consider now the case in which, in addition to the transmit power and the uplink linear receiver, the spreading code may also be tuned so as to maximize energy efficiency of the active users. Assuming that the spreading code is a unit-norm vector with real entries, the strategy space becomes

$$
\S_{k}=\left[0, P_{k, \max }\right] \times \mathcal{R}^{N} \times \mathcal{R}_{1}^{N},
$$

with $\mathcal{R}_{1}^{N}=\left\{\mathbf{d} \in \mathcal{R}^{N}: \mathbf{d}^{T} \mathbf{d}=1\right\}$ the set of allowable spreading codes for the $k$ th user. The associated maximization problem is now

$$
\max _{p_{k}, \mathbf{d}_{k}, \mathbf{s}_{k}} \frac{f\left(\gamma_{k}\left(p_{k}, \mathbf{d}_{k}, \mathbf{s}_{k}\right)\right)}{p_{k}}=\max _{p_{k}} \frac{f\left(\max _{\mathbf{d}_{k}, \mathbf{s}_{k}} \gamma_{k}\left(p_{k}, \mathbf{d}_{k}, \mathbf{s}_{k}\right)\right)}{p_{k}} .
$$

Based on (22), we can first take care of SINR maximization with respect to the spreading codes and linear receivers, and then focus on maximization of the resulting utility with respect to transmit power. In [10], it is shown that the game (22) admits a unique (uniqueness of the spreading code set is meant with respect to the set of eigenvalues of the matrix SHPH ${ }^{T} \mathbf{S}^{T}$ ) NE point through the following result.

Proposition 2. The noncooperative game defined in (22) admits a unique NE point $\left(p_{k}^{*}, \mathbf{d}_{k}^{*}, \mathbf{s}_{k}^{*}\right)$, for $k=1, \ldots, K$, where

(i) $\mathbf{s}_{k}^{*}$ and $\mathbf{d}_{k}^{*}$ are the unique fixed stable $k$ th user spreading code and receive filter (recall that the linear receive filter is unique up to a positive scaling factor) resulting from iterations

$$
\begin{aligned}
\mathbf{d}_{i} & =\sqrt{p_{i}} h_{i}\left(\mathbf{S H P H}^{T} \mathbf{S}^{T}+\frac{\mathcal{N}_{0}}{2} \mathbf{I}_{N}\right)^{-1} \mathbf{s}_{i}, \quad \forall i=1, \ldots, K, \\
\mathbf{s}_{i} & =\sqrt{p_{i}} h_{i}\left(p_{i} h_{i}^{2} \mathbf{D D}^{T}+\mu_{i} \mathbf{I}_{N}\right)^{+} \mathbf{d}_{i}, \quad \forall i=1, \ldots, K,
\end{aligned}
$$

with $\mu_{i}$ such that $\left\|\mathbf{s}_{i}\right\|^{2}=1, \mathbf{D}=\left[\mathbf{d}_{1}, \ldots, \mathbf{d}_{K}\right]$, and $(\cdot)^{+}$denoting generalized inverse. Denote by $\gamma_{k}^{*}$ the corresponding SINR. (ii) $p_{k}^{*}=\min \left\{\bar{p}_{k}, P_{k, \max }\right\}$, with $\bar{p}_{k}$ the kth user transmit power such that the $k$ th user maximum SINR $\gamma_{k}^{*}$ equals $\bar{\gamma}$, that is, the unique solution of the equation $f(\gamma)=$ $\gamma f^{\prime}(\gamma)$, where $f^{\prime}$ is the derivative of $f$.

Proof. The proof of this result is reported in [10]. Here, we give an alternative proof based on the theory of separable games [12]. Let $\mathbf{X}=(\mathbf{S}, \mathbf{D})$, and denote by $\mathbf{p}_{-k}=$ $\left[p_{1}, \ldots, p_{k-1}, p_{k+1}, \ldots, p_{K}\right]^{T}$ the $(K-1)$-dimensional vector containing the transmit powers of all the users except the $k$ th one. Upon defining

$$
I_{k}\left(\mathbf{X}, \mathbf{p}_{-k}\right)=\frac{1}{h_{k}^{2}\left(\mathbf{d}_{k}^{T} \mathbf{s}_{k}\right)^{2}}\left[\frac{\mathcal{N}_{0}}{2}\left\|\mathbf{d}_{k}\right\|^{2}+\sum_{i \neq k} p_{i} h_{i}^{2}\left(\mathbf{d}_{k}^{T} \mathbf{s}_{i}\right)^{2}\right],
$$

the utility function for the considered game can be written in the form

$$
u_{k}\left(p_{k}, I_{k}\left(\mathbf{X}, \mathbf{p}_{-k}\right)\right)
$$

Since, for fixed power $p_{k}$, the utility function is a decreasing function of $I_{k}$, the utility function (4) is said to be separable in the two parameters, $\mathbf{p}$ and $\mathbf{X}$ [12], and the corresponding game is a separable game. (Otherwise stated, in a separable game the utility function can be written as in (25), and for any fixed $p_{k}$, the utility function is a decreasing function of $I_{k}$.) Let us denote by $g_{X}(\mathbf{p})$ and by $g_{p}(\mathbf{X})$ the subgame arising from utility maximization with respect to the spreading code and uplink linear receiver optimization for a fixed transmit power configuration $\mathbf{p}$, and the subgame arising from utility maximization with respect to transmit power for fixed spreading codes and linear receivers, respectively. Based on the results of $[16,17]$, it can be shown that the subgame $g_{X}\left(\mathbf{p}^{*}\right)$ admits a unique $\mathrm{NE}$ point $\mathbf{X}^{*}=\left(\mathbf{S}^{*}, \mathbf{D}^{*}\right)$, arising from the unique stable fixed point of iterations (23); (see also [18] for a proof of the convergence properties of iterations (23).) Similarly, for any user $k$, the transmit power $p_{k}^{*}$, as defined in the text of Proposition 2, is an NE point for the subgame $g_{p}\left(\mathbf{S}^{*}, \mathbf{D}^{*}\right)[7]$.

Given any interference $I_{k}\left(\mathbf{X}, \mathbf{p}_{-k}\right)$, the power $p_{k}^{*}=$ $\min \left(\bar{\gamma} I_{k}\left(\mathbf{X}, \mathbf{p}_{-k}\right), P_{k, \max }\right)$ maximizes the utility of the user $k$ and it is a continuous function of the interference $I_{k}$. Moreover, it is easily seen that denoting by $\mathbf{X}^{*}(\mathbf{p})$ the NE of the subgame $g_{X}(\mathbf{p})$, the interference function $I_{k}\left(\mathbf{X}^{*}(\mathbf{p}), \mathbf{p}_{-k}\right)$ in (24) is continuous in $\mathbf{p}$, for $k=1, \ldots, K$. According thus to [12, Theorem 2], the existence of an NE for game (22) is guaranteed. Additionally, since the subgames $g_{X}(\mathbf{p})$ and $g_{p}(\mathbf{X})$ have unique NE points, the uniqueness of the NE for the considered game is also proved.

This NE can be reached alternating between these two phases.

(a) Given the transmit powers $\mathbf{p}$, each user adjusts its spreading code and receives filter through iterations (23) until an equilibrium is reached.

(b) Given the spreading codes and uplink receivers, each user tunes its transmit power so that its own SINR equals $\bar{\gamma}$, 
according to the iterations

$$
\begin{aligned}
p_{k}= & \frac{\bar{\gamma}}{h_{k}^{2}\left(\mathbf{d}_{k}^{T} \mathbf{s}_{k}\right)^{2}} \\
& \times\left[\frac{\mathcal{N}_{0}}{2}\left\|\mathbf{d}_{k}\right\|^{2}+\sum_{i \neq k} p_{i} h_{i}^{2}\left(\mathbf{d}_{k}^{T} \mathbf{s}_{i}\right)^{2}\right], \quad \forall k=1, \ldots, K .
\end{aligned}
$$

Steps $\mathrm{a}$ and $\mathrm{b}$ are repeated until convergence is reached. Since $I_{k}\left(\mathbf{X}, \mathbf{p}_{-k}\right)$ is an increasing function of $\mathbf{p}_{-k}$, convergence of this procedure is guaranteed to hold. Note also that, for the case in which the number of users does not exceed the processing gain, the NE point is shown in [10] to be also Pareto-optimal.

Also in this case, nonadaptive implementation of the considered game requires knowledge of several parameters of the interference, thus implying that, despite its noncooperative nature, the game, in its current form, is not amenable to a decentralized implementation. This problem is circumvented in the following, where an adaptive procedure is proposed enabling fully decentralized game playing. Again we assume here knowledge of the channel for the user of interest. First of all, we note that, as discussed in [16], iterations (23) converge to the global minimum of the total mean square error (TMSE) of the system, which is defined as the sum of the mean square error (MSE) for all the active users; moreover, according to [17], it can be shown that, in the considered scenario of single-path fading channel, minimization of the TMSE is equivalent to selfish noncooperative minimization of the MSE of each user with respect to its spreading code and linear receiver. As a consequence, we can substitute, with no effect on the equilibrium solution, iterations (23) with new ones where each user updates its own spreading code and receives filter aiming at selfish minimization of its own MSE. The $k$ th user's MSE can be written as

$$
\operatorname{MSE}_{k}=1+\mathbf{d}_{k}^{T}\left(p_{k} h_{k}^{2} \mathbf{s}_{k} \mathbf{s}_{k}^{T}+\mathbf{R}_{k}\right) \mathbf{d}_{k}-2 \sqrt{p_{k}} h_{k} \mathbf{d}_{k}^{T} \mathbf{s}_{k},
$$

with $\mathbf{R}_{k}=\mathbf{R}-p_{k} h_{k}^{2} \mathbf{s}_{k} \mathbf{s}_{k}^{T}$, the covariance matrix of the interference that affects the $k$ th user. Minimization of (27) with respect to $\mathbf{d}_{k}$ and $\boldsymbol{s}_{k}$, under the constraint $\left\|\boldsymbol{s}_{k}\right\|^{2}=1$, yields the following iterations (see also [19]):

$$
\begin{aligned}
\mathbf{d}_{i} & =\sqrt{p_{i}} h_{i}\left(\mathbf{S H P H}^{T} \mathbf{S}^{T}+\frac{\mathcal{N}_{0}}{2} \mathbf{I}_{N}\right)^{-1} \mathbf{s}_{i}, \quad \forall i=1, \ldots, K, \\
\mathbf{s}_{i} & =\frac{\mathbf{d}_{i}}{\left\|\mathbf{d}_{i}\right\|}, \quad \forall i=1, \ldots, K .
\end{aligned}
$$

The fixed points of iterations (28) coincide with those of iterations (23) [17]; however, despite such equivalence, the spreading code update for each user in (28) amounts to a normalized scaling of its own MMSE receiver, thus implying that the result of the already outlined adaptive RLS-based implementation of the receiver (or, alternatively, of the blind LMS update) may be used also for the spreading code update. The stochastic implementation of the joint power vector, spreading code and receive filter optimization algorithm, is thus the following. After the receive filter update (12), the $k$ th user can optimize its own spreading sequence according to $\mathbf{s}_{k}(n)=\mathbf{d}_{k}(n) /\left\|\mathbf{d}_{k}(n)\right\|$; then, the stochastic power control update (16) can be computed. When testing such a procedure through numerical simulations, it has been noted that faster and smoother convergence occurs when at each iteration only one user updates its spreading code, that is, spreading codes are to be updated cyclically, thus implying that each user, after the update of its own spreading code, will not be updating it for a certain number of symbol intervals. Summing up, we propose an adaptive algorithm based on the following steps.

(1) Set $\hat{\mathbf{R}}(0)=\epsilon \mathbf{I}_{N}, \mathbf{d}_{k}(0)=\boldsymbol{s}_{k}$ and $p_{k}(0)=$ $P_{k, \max } / 2, \forall k=1, \ldots, K$. Set $n=0$ and $\ell=0$.

(2) Set $n=n+1$.

(3) Update $\mathbf{d}_{k}(n)$ using either the RLS iterations (12) or the blind LMS iteration (14), for $k=1, \ldots, K$.

(4) Set $\ell=\ell+1$, and $m=(\ell \bmod K)+1$; compute $\mathbf{s}_{m}(n)=\mathbf{d}_{m}(n) /\left\|\mathbf{d}_{m}(n)\right\|$ and send it back to the user terminal.

(5) Compute $\ell_{k}(n)$ using (17), for any $k=1, \ldots, K$, and send it back to the user terminals.

(6) The user terminals can now update their transmit powers based on (16).

(7) Go to step 2.

Note that, in each symbol interval, the receive filter and the transmit power are updated for every user, while the spreading code is updated for one user only.

\section{Cross-Layer Resource Allocation in Single-Cell Networks: Unknown Channel}

Consider now the more realistic case in which each user must estimate its own channel coefficient based on a training sequence, and uses this estimate in the successive distributed implementation of the game. In particular, assume that, for each user $k$, the data $b_{k}(0), \ldots, b_{k}(T-1)$ are training symbols (with $T$ the length of the training sequence) to be used for estimating the channel coefficients. As far as channel estimation is concerned, it is more convenient to rewrite the data vector $\mathbf{r}(n)$ in the form

$$
\mathbf{r}(n)=\mathbf{S}(n) \mathbf{P}^{1 / 2}(n) \mathbf{B}(n) \mathbf{h}+\mathbf{w}(n),
$$

where $\mathbf{B}(n)$ is a $K \times K$-dimensional diagonal matrix, whose diagonal is $\left[b_{1}(n), \ldots, b_{K}(n)\right]$, and $\mathbf{h}=\left[h_{1}, \ldots, h_{K}\right]^{T}$ is the $K$-dimensional vector of the channel gains.

A first strategy is to resort to maximum likelihood (ML) channel estimation. Conditioned upon the known training symbols, the probability distribution of the data vectors $\mathbf{r}(0), \ldots, \mathbf{r}(T-1)$ is Gaussian, thus implying that the ML channel estimate is

$$
\widehat{\mathbf{h}}=\arg \min _{\mathbf{h}} \sum_{n=0}^{T-1}\left\|\mathbf{r}(n)-\mathbf{S}(n) \mathbf{P}^{1 / 2}(n) \mathbf{B}(n) \mathbf{h}\right\|^{2},
$$


whose solution is

$$
\begin{aligned}
\widehat{\mathbf{h}}=[ & \left.\sum_{n=0}^{T-1} \mathbf{B}(n) \mathbf{P}^{1 / 2}(n) \mathbf{S}^{T}(n) \mathbf{S}(n) \mathbf{P}^{1 / 2}(n) \mathbf{B}(n)\right]^{-1} \\
& \times\left[\sum_{n=0}^{T-1} \mathbf{B}(n) \mathbf{P}^{1 / 2}(n) \mathbf{S}^{T}(n) \mathbf{r}(n)\right] .
\end{aligned}
$$

It is worth noting that (31) requires the inversion of a $K \times$ $K$-dimensional matrix; however, lower-complexity solutions based on the steepest descent rule can be also devised, such as

$$
\begin{aligned}
\widehat{\mathbf{h}}(n) & \\
= & {\left[\mathbf{I}_{K}-\alpha \sum_{j=0}^{n} \mathbf{B}(j) \mathbf{P}^{1 / 2}(j) \mathbf{S}^{T}(j) \mathbf{S}(j) \mathbf{P}^{1 / 2}(j) \mathbf{B}(j)\right] \widehat{\mathbf{h}}(n-1) } \\
& +\alpha\left[\sum_{j=0}^{n} \mathbf{B}(j) \mathbf{P}^{1 / 2}(j) \mathbf{S}^{T}(j) \mathbf{r}(j)\right]
\end{aligned}
$$

where the step-size $\alpha$ is a close-to-zero positive constant.

Another strategy is instead based on a Bayesian approach, that is, the use of MMSE channel estimation. In order to implement the MMSE channel estimate $\hat{\mathbf{h}}$ iteratively, we can use an MMSE sequential estimator such as the Kalman filter $[13,20]$. Note that the data are now expressed as

$$
\begin{aligned}
\mathbf{r}(n) & =\mathbf{S}(n) \mathbf{P}^{1 / 2}(n) \mathbf{B}(n) \mathbf{h}(n)+\mathbf{w}(n) \\
& =\mathbf{A}(n) \mathbf{h}(n)+\mathbf{w}(n),
\end{aligned}
$$

where we let $\mathbf{A}=\mathbf{S P}^{1 / 2} \mathbf{B}$. Since we are considering a slowly fading channel, the state update equation for our statespace model is simply $\mathbf{h}(n)=\mathbf{h}(n-1)$. Consequently, the prediction equation reduces to

$$
\widehat{\mathbf{h}}(n \mid n-1)=\widehat{\mathbf{h}}(n-1 \mid n-1),
$$

where $\hat{\mathbf{h}}(n \mid m)$ denotes the estimate of $\mathbf{h}(n)$ based on the observations $[\mathbf{r}(0), \mathbf{r}(1), \ldots, \mathbf{r}(m)]$. Similarly, the matrix denoting the minimum prediction error $\mathbf{Q}$ has the following update equation:

$$
\mathbf{Q}(n \mid n-1)=\mathbf{Q}(n-1 \mid n-1),
$$

and the vector Kalman filter for the channel vector estimation reduces to these equations:

$$
\begin{aligned}
& \mathbf{K}(n)=\mathbf{Q}(n-1) \mathbf{A}^{T}(n)\left[\frac{\mathcal{N}_{0}}{2} \mathbf{I}_{K}+\mathbf{A}(n) \mathbf{Q}(n-1) \mathbf{A}^{T}(n)\right]^{-1}, \\
& \mathbf{Q}(n)=\left[\mathbf{I}_{K}-\mathbf{K}(n) \mathbf{A}(n)\right] \mathbf{Q}(n-1), \\
& \widehat{\mathbf{h}}(n)=\widehat{\mathbf{h}}(n-1)+\mathbf{K}(n)[\mathbf{r}(n)-\mathbf{A}(n) \hat{\mathbf{h}}(n-1)],
\end{aligned}
$$

where the $K \times N$-dimensional matrix $\mathbf{K}(n)$ is the Kalman gain matrix, and the $K \times K$-dimensional matrix $\mathbf{Q}(n)$ is the minimum MSE matrix for the $n$th symbol interval.
Either of the above estimation strategies provides a good estimate of the channel vector $\hat{\mathbf{h}}$, which can be used during the implementation of the stochastic algorithms proposed in the previous section. So, to take into account the channel estimation phase, the algorithms are modified as follows. First, the SINR target $\bar{\gamma}$ is determined by solving the equation $f(\gamma)=\gamma f^{\prime}(\gamma)$. Then, during the training phase $(n \leq T)$, each user computes only its own receive filter, applying the RLS algorithm (12), and the channel vector estimate, while a centralized processor computes the channel estimates either via the ML adaptive algorithm (32) or the Kalman filter (36). For $n>T$, the following phases are then performed.

(1a) Given the transmit power vector $\mathbf{p}(n)$, the AP computes the receive filter for each user applying either the RLS algorithm (12) or the blind LMS algorithm (14).

(1b) For the joint power control, receive filter, and spreading code optimization game only, each user also cyclically updates its own spreading sequence according to the second line of (28).

(2) Given its own estimated channel coefficient and the updated receive filter and spreading code, each user implements the stochastic power control algorithm (16).

\section{Cross-Layer Resource Allocation in Multicell Networks}

Let us now extend our analysis to a multicell environment, in which each user is affected by interference from users' terminals outside its own cell in addition to the interference of the ones within the same cell. When an AP is designated to detect the received signal from a given user's transmitter, we say that the user has been assigned to that AP. The AP assignment is denoted by the $K$-dimensional vector $\mathbf{a}=$ $\left(a_{1}, \ldots, a_{K}\right)$, whose entry $a_{i} \in\{1, \ldots, B\}$. We can note that there are $B^{K}$ different assignments possible. The AP assignment problem is beyond the scope of this paper, and indeed we assume here that the AP assignment vector a has been determined in a previous phase and we focus on the resource allocation problem only. In a previous work [21], the game-theoretic approach has been applied to the power control problem in multicell networks, using conventional matched filters at the receiver for every user. The authors have formulated the corresponding noncooperative game and have demonstrated the existence and uniqueness of an NE point. Some further results are then reported in [10], where the problem of joint power control and linear receiver choice is addressed, and additionally the case of spreading code allocation is also briefly treated.

The received data vector, in the $j$ th $\mathrm{AP}$ and at the $n$th epoch, can now be written as follows:

$$
\mathbf{r}_{j}(n)=\sum_{k=1}^{K} \sqrt{p_{k}}(n) h_{j, k} b_{k}(n) \mathbf{s}_{k}(n)+\mathbf{w}_{j}(n) \quad \forall j=1, \ldots, B,
$$


where $h_{j, k}$ is the channel gain between the $k$ th user's transmitter and the $j$ th AP. A more compact representation of (37) is given by

$$
\mathbf{r}_{j}(n)=\mathbf{S}(n) \mathbf{P}^{1 / 2}(n) \mathbf{H}_{j} \mathbf{b}(n)+\mathbf{w}_{j}(n) \quad \forall j=1, \ldots, B,
$$

where $\mathbf{H}_{j}$ is a $K \times K$-dimensional diagonal matrix whose diagonal is $\left[h_{j, 1}, \ldots, h_{j, K}\right]$, that is, the channel gains between the $j$ th AP and all the users in the network.

Denoting by $\gamma_{j, k}$ the $k$ th user's SINR at the $j$ th AP, the utility function to be maximized can now be expressed as

$$
u_{k}=R \frac{L}{M} \frac{f\left(\gamma_{a_{k}, k}\right)}{p_{k}}, \quad \forall k=1, \ldots, K,
$$

where $\gamma_{a_{k}, k}$ is the SINR achieved by the $k$ th user at its assigned AP. Similarly to the single-cell case, we are now interested in formulating stochastic implementations of noncooperative resource allocation games.

5.1. Cross-Layer Power Allocation and Receiver Choice. The following result is reported in [10].

Proposition 3. Consider a noncooperative game where the $k$ th user's utility (39) is maximized with respect to the choice of the transmit power $p_{k} \in\left[0, P_{k, \max }\right]$ and of the linear receiver $\mathbf{d}_{k} \in \mathcal{R}^{N}$. A unique $N E$ point $\left(p_{k}^{*}, \mathbf{d}_{k}^{*}\right)$, for $k=1, \ldots, K$, exists, where

(i) $\mathbf{d}_{k}^{*}$ is the vector corresponding to a linear MMSE receiver; and

(ii) $p_{k}^{*}=\min \left\{\bar{p}_{k}, P_{k, \max }\right\}$, with $\bar{p}_{k}$ the $k$ th user's transmit power such that the kth user's SINR $\gamma_{a_{k}, k}$ equals $\bar{\gamma}$, that is, the unique solution of the equation $f(\gamma)=\gamma f^{\prime}(\gamma)$, where $f^{\prime}$ is the derivative of $f$.

It can be easily seen that this result is quite similar to that stated in Proposition 1, the only difference being the fact that the $k$ th user's symbols are decoded based on the data received at the $a_{k}$ th AP. As a consequence, the $k$ th user's MMSE receiver is $\mathbf{d}_{k}(n)=$ $\sqrt{p_{k}(n)} h_{a_{k}, k}\left(\mathbf{S}(n) \mathbf{H}_{a_{k}} \mathbf{P}(n) \mathbf{H}_{a_{k}}^{T}(n) \mathbf{S}^{T}(n)+\left(\mathcal{N}_{0} / 2\right) \mathbf{I}_{N}\right)^{-1} \boldsymbol{s}_{k}(n)$.

As a consequence, a stochastic implementation of this game requires the following: (a) implementation of the adaptive MMSE receiver according to either (12) or (14), and replacing $\mathbf{r}(n)$ with $\mathbf{r}_{a_{k}}(n)$; and (b) implementation of the power control update based on (16), provided that in (17) the quantities $\mathbf{r}(n)$ and $h_{k}$ are replaced by $\mathbf{r}_{a_{k}}(n)$ and $h_{a_{k}, k}$, respectively. Formally, the steps of the algorithm are as follows.

(1) Set $\widehat{\mathbf{R}}(0)=\epsilon \mathbf{I}_{N} ; \mathbf{d}_{k}=\mathbf{s}_{k}$ and $p_{k}(0)=P_{k, \max } / 2, \forall k=$ $1, \ldots, K$. Set $n=0$ and $\ell=0$.

(2) Set $n=n+1$.

(3) Update $\mathbf{d}_{k}(n)$ using either the RLS iterations (12) or the blind LMS iteration (14), provided that $\mathbf{r}(n)$ is replaced by $\mathbf{r}_{a_{k}}(n)$, for $k=1, \ldots, K$.
(4) Compute $\ell_{k}(n)$ according to

$$
\begin{aligned}
\ell_{k}(n)= & \frac{\bar{\gamma}}{h_{a_{k}, k}^{2}\left(\mathbf{d}_{k}^{T}(n) \mathbf{s}_{k}(n)\right)^{2}} \\
& \times\left[\left(\mathbf{d}_{k}^{T}(n) \mathbf{r}_{a_{k}}(n)\right)^{2}-p_{k}(n) h_{a_{k}, k}^{2}\left(\mathbf{d}_{k}^{T}(n) \mathbf{s}_{k}(n)\right)^{2}\right],
\end{aligned}
$$

$\forall k=1, \ldots, K$, and send it back to the user terminal.

(5) The user terminals can now update their transmit powers based on (16).

(6) Go to step 2.

Regarding the convergence of this algorithm, also for this case, the stochastic interference function can be easily shown to be quasistandard [15], thus implying that the theoretical convergence results of Section 3.2 can be extended straightforwardly to the multicell scenario as well. Otherwise stated, we are able to prove almost sure convergence of the transmit power vector to the equilibrium vector of the nonadaptive game if a sufficiently large number of receiver updates are performed between two consecutive power updates. For the sake of conciseness, we do not give here further details, since a thorough extension to the multicell setting of the results of Section 3.2 would not add much conceptual value to the paper. The numerical simulations, to be illustrated in the sequel of the paper, will prove the effectiveness of the proposed algorithm.

5.2. Cross-Layer Power, Spreading Code and Receiver Allocation. Consider now the case in which utility (39) is maximized with respect to the spreading code, transmit power and linear receiver choice. In this case, no theoretical results are available on the existence of an $\mathrm{NE}$ for the considered game. Indeed, first of all, note that

$$
\max _{\mathbf{s}_{k}, \mathbf{d}_{k}, p_{k}} \frac{f\left(\gamma_{a_{k}, k}\right)}{p_{k}}=\max _{p_{k}} \frac{f\left(\max _{\boldsymbol{s}_{k}, \mathbf{d}_{k}} \gamma_{a_{k}, k}\left(p_{k}, \mathbf{s}_{k}, \mathbf{d}_{k}\right)\right)}{p_{k}},
$$

thus implying that we must consider first SINR maximization with respect to the spreading code and linear receiver choice, and then utility maximization with respect to the transmit power $p_{k}$. Omitting, for the sake of brevity, the mathematical details, it can be shown that playing the noncooperative SINR maximization game is tantamount to implementing the iterations

$$
\begin{aligned}
\mathbf{d}_{i}(n)= & \sqrt{p_{i}(n)} h_{a_{i},}\left(\mathbf{S}(n) \mathbf{H}_{a_{i}} \mathbf{P}(n) \mathbf{H}_{a_{i}}^{T}(n) \mathbf{S}^{T}(n)+\frac{\mathcal{N}_{0}}{2} \mathbf{I}_{N}\right)^{-1} \\
& \times \mathbf{s}_{i}(n), \quad \forall i=1, \ldots, K, \\
\mathbf{s}_{i}(n)= & \frac{\mathbf{d}_{i}(n)}{\left\|\mathbf{d}_{i}(n)\right\|}, \quad \forall i=1, \ldots, K .
\end{aligned}
$$

While in a single-cell scenario the noncooperative SINR maximization game admits an $\mathrm{NE}$ (see $[10,16])$, in a multicell system, such an equilibrium is not guaranteed 


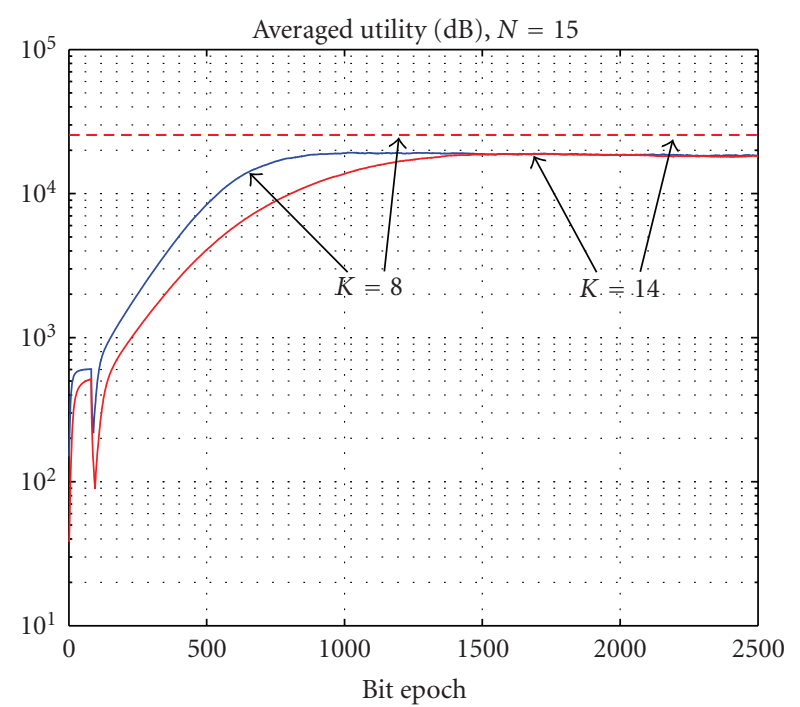

$\begin{array}{llll}\text { - Achieved utility ( } 8 \text { users) } & --- & \text { Target utility ( } 8 \text { users) } \\ \text { - Achieved utility (14 users) } & --- & \text { Target utility (14 users) }\end{array}$

Figure 2: Achieved average utility versus time. The system processing gain is $N=15$. We report here the performance of the algorithm presented in Section 3.3. The horizontal lines represent the equilibrium performance of the nonadaptive implementation of the games.

to exist. Otherwise stated, due to the fact that the same user is received with different powers at different APs, iterations (42) cannot be shown to have (possibly stable) fixed points, and indeed through numerical simulations we found many instances where no equilibrium is reached. On the other hand, our own simulation trials have also shown that implementation of this game, although not leading to an equilibrium point, generally permits finding spreading codes sets that have a better performance (in terms of achieved energy efficiency) than the original set of spreading codes, thus implying that running equations (42) for a certain number of iterations is beneficial to the system performance, even when no NE can be reached. Based on these arguments, it is thus of interest to provide an adaptive procedure for energy efficiency maximization in this case too. The procedure that we propose is thus the following: (a) implementation of the adaptive MMSE receiver according to either (12) or (14), and replacing $\mathbf{r}(n)$ with $\mathbf{r}_{a_{k}}(n)$; (b) implementation of the spreading code update equation $\mathbf{s}_{k}(n)=\mathbf{d}_{k}(n) /\left\|\mathbf{d}_{k}(n)\right\|$, this step is to be performed cyclically among users, that is, at each symbol epoch only one user is to update its own spreading code; and (c) implementation of the power control update based on (16), provided that in (17) the quantities $\mathbf{r}(n)$ and $h_{k}$ are replaced by $\mathbf{r}_{a_{k}}(n)$ and $h_{a_{k}, k}$, respectively.

Although, as already discussed, this procedure is not guaranteed to converge to an NE even in the nonadaptive case, numerical results, as illustrated in the following section, will show that it helps in obtaining a better-performing set of spreading codes.

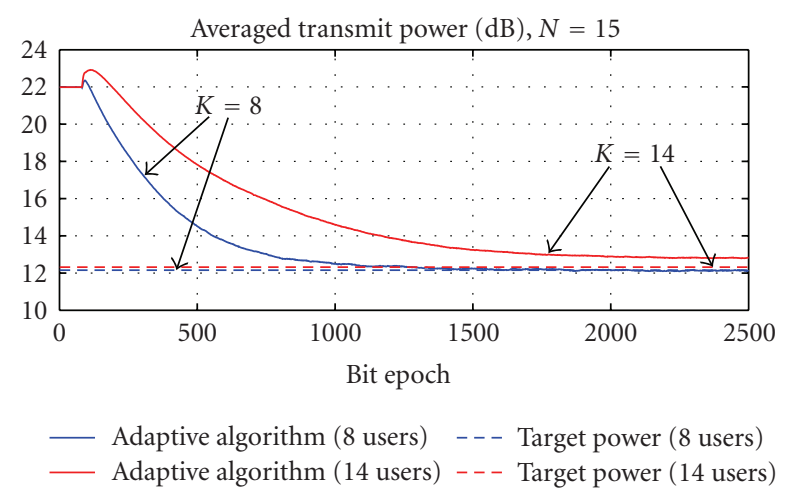

(a)

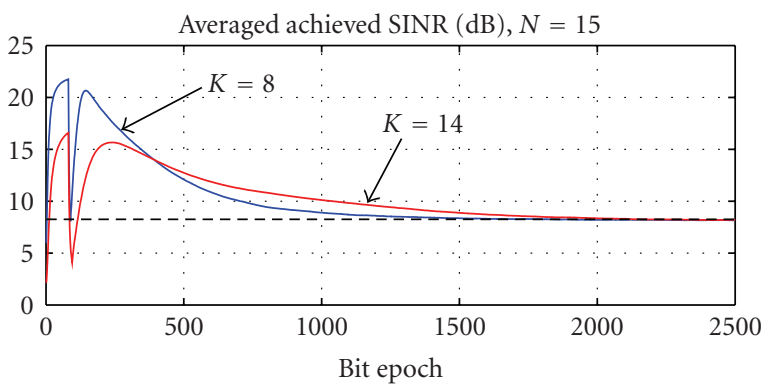

$\begin{array}{ll}\text { — } & \text { Achieved SINR (8 users) } \\ \text { - } & \text { Achieved SINR (14 users) } \\ \text { - - } & \text { Target SINR }\end{array}$

(b)

Figure 3: Achieved average SINR and power versus time. The system processing gain is $N=15$. We report here the performance of the algorithm presented in Section 3.3. The horizontal lines represent the equilibrium performance of the nonadaptive implementation of the games.

\section{Numerical Results}

We contrast here the performance of the deterministic algorithms of the noncooperative games with their adaptive implementations proposed in this paper. We start by considering an uplink DS/CDMA system with processing gain $N=15$, and assume that the packet length is $M=120$. For this value of $M$, the equation $f(\gamma)=\gamma f^{\prime}(\gamma)$ can be shown to admit the solution $\bar{\gamma}=6.689=8.25 \mathrm{~dB}$. A single-cell system is first considered, where the channel coefficient $h_{k}$ for the generic $k$ th user, is assumed to be Rayleigh distributed with mean equal to $-20 \mathrm{~dB}$. We take the ambient noise level to be $\mathcal{N}_{0}=10^{-2} \mathrm{~W} / \mathrm{Hz}$, while the maximum allowed power $P_{k, \max }$ is $25 \mathrm{~dB}$. We present the results of averaging over 500 independent realizations for the users' fading channel coefficients and starting set of spreading codes. More precisely, for each iteration, we randomly generate an $N \times K$ dimensional spreading code matrix with entries in the set $\{-1 / \sqrt{N}, 1 / \sqrt{N}\}$; this matrix is then used as the starting point for the joint power control, receive filter, and spreading code optimization games. 


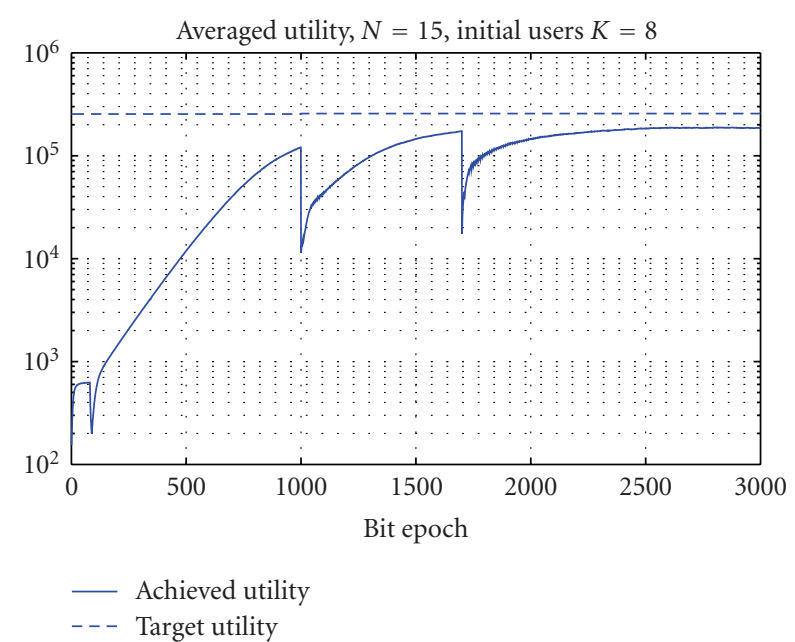

(a)

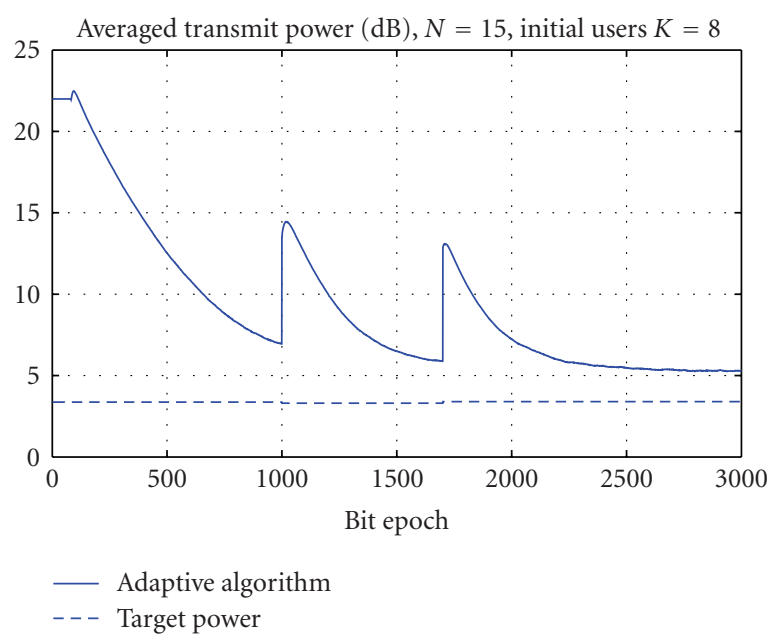

(b)

FIgURe 4: Achieved average utility and power versus time for the algorithm presented in Section 3.3. The initial number of users is $K=8$, while two additional users enter the channel at bit epochs 1000 and 1700 .

Figures 2-3 compare the behavior of the adaptive algorithm for the joint power control, receive filter, and spreading code optimization with its nonadaptive version. We assume that an RLS algorithm is used in step 3 of the algorithm presented at the end of Section 3.3, with $T=80$ training symbols, while in (16) the step size $\rho$ equals 0.01 . Figures 2-3 report the time-evolution of the achieved average utility (measured in bit/Joule), the average achieved SINR, and the average transmit power, respectively, for $K=8$ and $K=$ 14. It is seen that after about one thousand iterations, the adaptive algorithm approximates with satisfactory accuracy the benchmark scenario of a nonadaptive game as in [10]. In particular, while the target SINR and the achieved utility are quite close to their target values, it is seen from Figure 3 that the average transmit power is about $3 \mathrm{~dB}$ larger than in the nonadaptive case; such a loss is not at all surprising, since

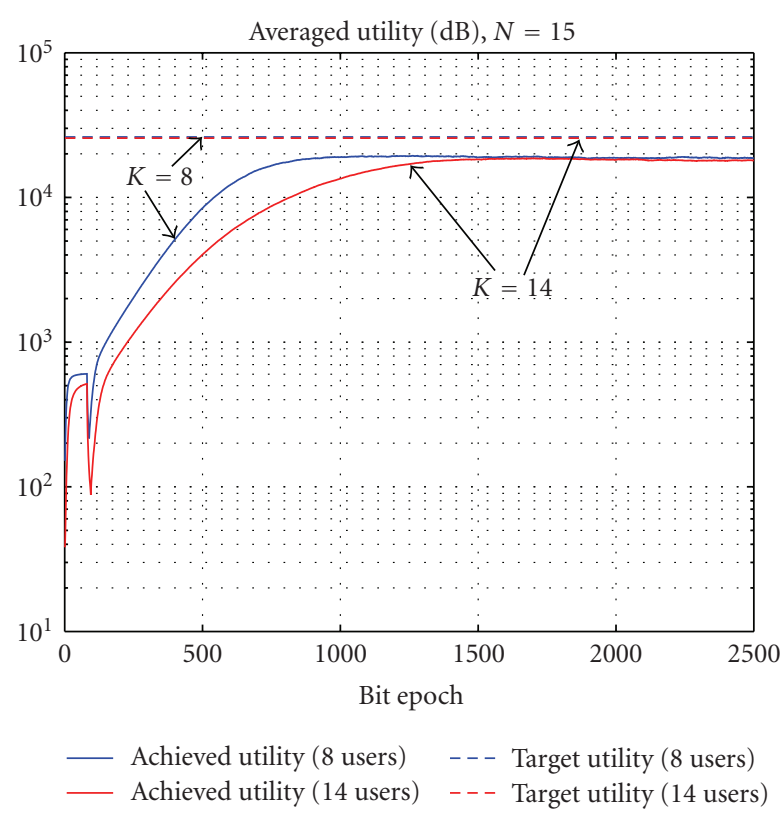

FIGURE 5: ML channel estimation. Average achieved utility versus time.

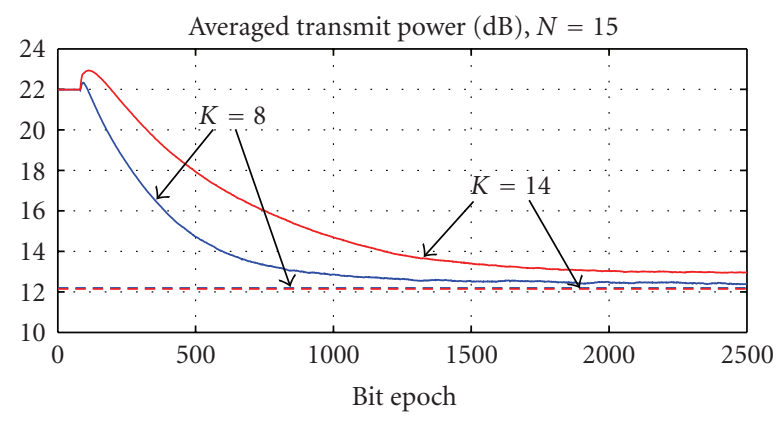

- Adaptive algorithm (8 users) - - - Target power (8 users)

— Adaptive algorithm (14 users) - - - Target power (14 users)

(a)

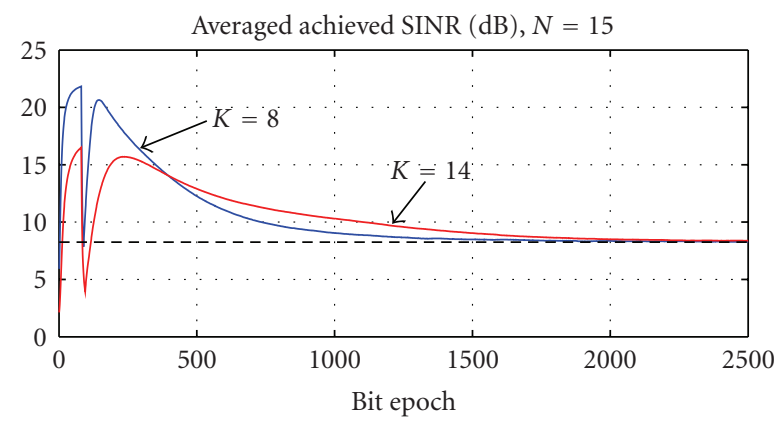

— Achieved SINR (8 users)
— - Achieved SINR (14 users)
Target SINR

(b)

FIGURE 6: ML channel estimation. Achieved average SINR and power versus time. 


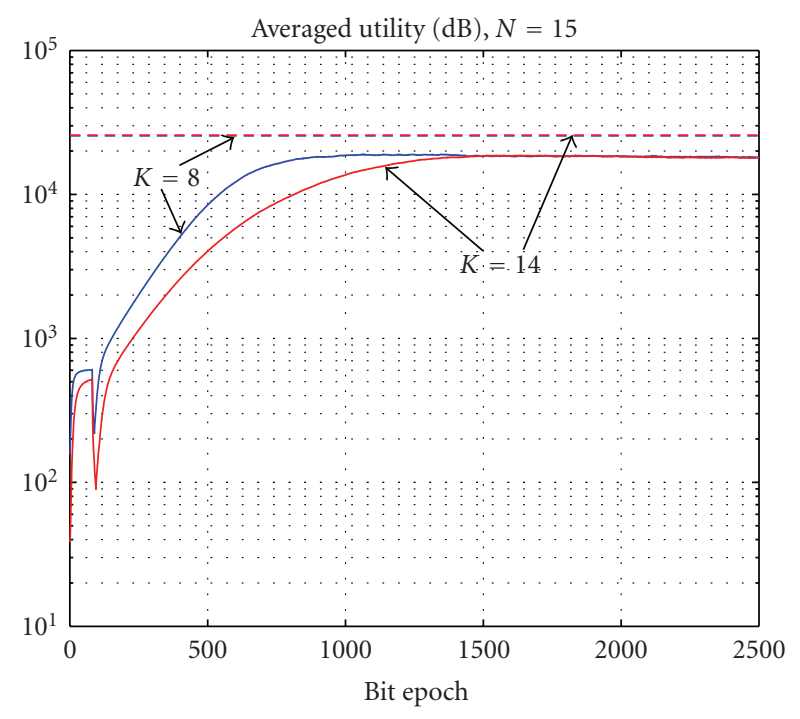

_ Achieved utility ( 8 users) - - - Target utility (8 users)

— Achieved utility (14 users) - - - Target utility (14 users)

Figure 7: MMSE (Kalman-based) channel estimation. Average achieved utility versus time.

it is well-known that adaptive algorithms have a steady-state error, and that their performance may only approach that of their nonadaptive counterparts.

In order to test the tracking properties of the proposed algorithm, we also consider a dynamic scenario in which the number of users is changing. We consider a system with an initial number of users $K=8$, and with two additional users entering the channel at time epochs $n=1000$ and $n=1700$. The results are reported in Figure 4 for both the average utility and power. These results clearly show that the algorithm is capable of coping with changes in the interference background.

Consider now the case in which the channel coefficients are to be estimated based on the training symbols. Figures 5-6 report the results concerning the application of the ML criterion, both with $K=8$ and $K=14$, where the stepsize used is $\alpha(n)=\tilde{\alpha} / n$, with $\tilde{\alpha}=10^{-4}$. In Figure 7 results concerning the application of the Kalman filter are reported. The simulation parameters are the same as those of Figures 5 and 6. It is seen that the achieved performance is quite similar to that obtained for the case of ML channel estimation.

Consider now a multicell environment, wherein $B=3$ APs are distributed in a square area with side $L=1000 \mathrm{~m}$. The considered system processing gain is now $N=10$; we present simulation results for number of users $K=15$ and $K=24$ (this latter case corresponds to an average system load per cell equal to 0.8 ). The $K$ active users are randomly distributed in the area, with a $10 \mathrm{~m}$ minimum distance from each AP. The channel coefficient $h_{j, k}$ is assumed to be Rayleigh distributed with mean equal to $d_{j k}^{-2}$, with $d_{j k}$ being the distance of user $k$ from the base station $j$. Here we assume that each user is assigned to its closest AP. The ambient noise level is assumed to be $\mathcal{N}_{0}=10^{-5} \mathrm{~W} / \mathrm{Hz}$. The training

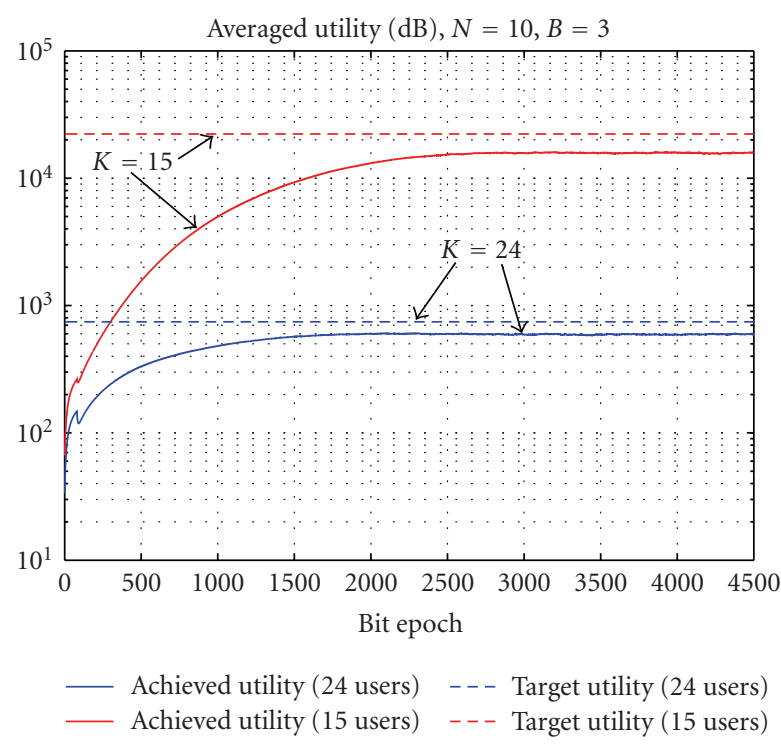

FIGURE 8: Multicell environment. Average utility versus time for the algorithm presented in Section 5.1. The horizontal lines represent the equilibrium performance of the nonadaptive implementation of the games.

phase has a length of $T=80$ bits. Figures 8-9 compare the behavior of the adaptive joint power control and receive filter optimization with its nonadaptive version. Also in this case, it is seen that the adaptive algorithms approach with good accuracy the performance of their nonadaptive counterparts, although in the multicell scenario convergence speed may be slower than in the single-cell case.

Finally, in Figure 10 we give numerical evidence of the beneficial effect that optimization with respect to the spreading codes set has in a multicell environment. We consider a system with $B=4, N=20$, and $K=30$. Here, we report the achieved utility and the average transmit power versus time for stochastic implementations of the resource allocation games of Section 5, with and without spreading code optimization. It is seen that, despite the lack of an NE point, optimization with respect to the spreading codes set brings noticeable performance improvements both in terms of larger energy efficiency and of smaller transmit power.

\section{Conclusions}

In this paper, the problem of adaptive cross-layer resource allocation in wireless data networks has been considered. In particular, adaptive algorithms for distributed energy efficiency maximization in a noncooperative context have been proposed. The resources to be allocated are the transmit power, the linear uplink receiver, and the spreading code of each active user in the network. Both single-cell and multicell networks have been considered. The performance and convergence properties of the proposed algorithms have been analyzed through some theoretical considerations, and more extensively through numerical experiments. Results have shown that the outlined solutions are effective and are 


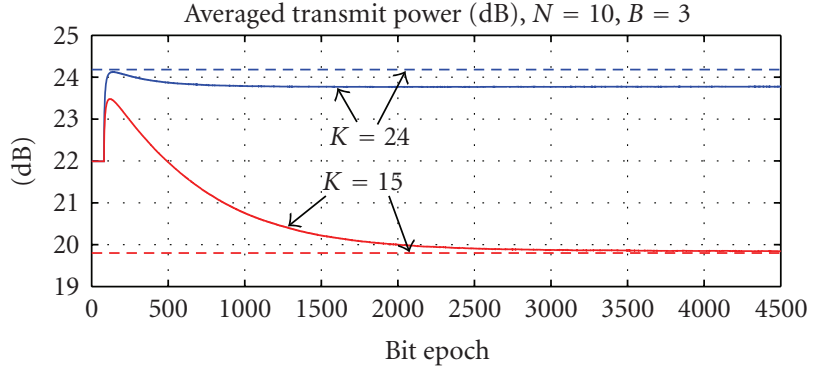

$\begin{array}{lll}\text { — Adaptive algorithm (24 users) } & --- \text { Target power (24 users) } \\ \text { Adaptive algorithm (15 users) } & --- \text { Target power (15 users) }\end{array}$

(a)

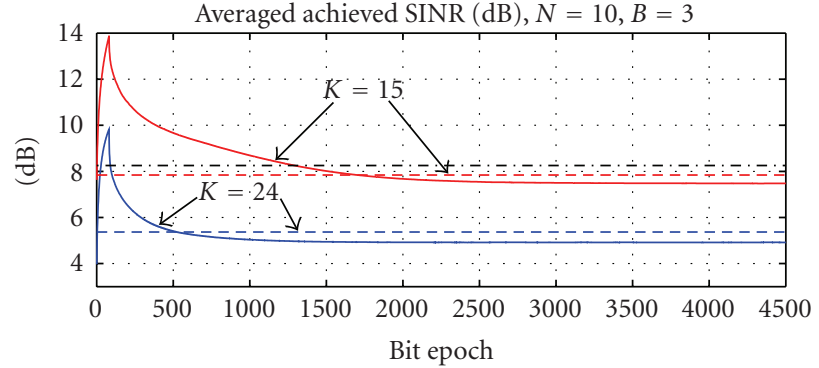

— Achieved SINR (24 users) $\quad---$ Deterministic SINR (15 users)
_ Achieved SINR (15 users) - - - Target SINR
- Deterministic SINR (24 users)

(b)

FIgure 9: Multicell environment. Achieved average SINR and power versus time for the algorithm presented in Section 5.1. The horizontal lines represent the equilibrium performance of the nonadaptive implementation of the games.

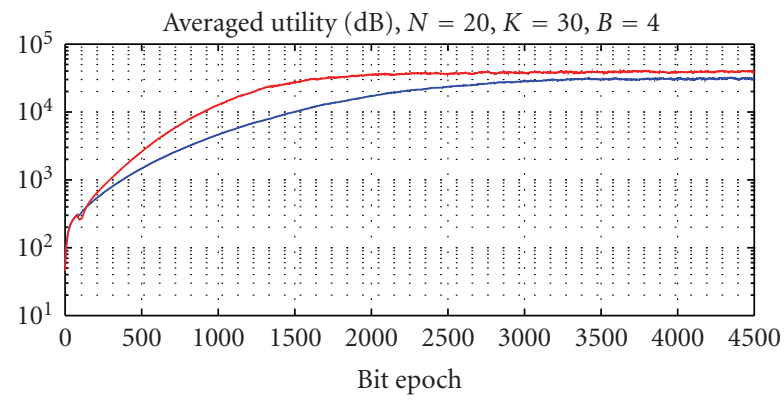

Achieved utility (without sequence update)
Achieved utility (with sequence update)

(a)

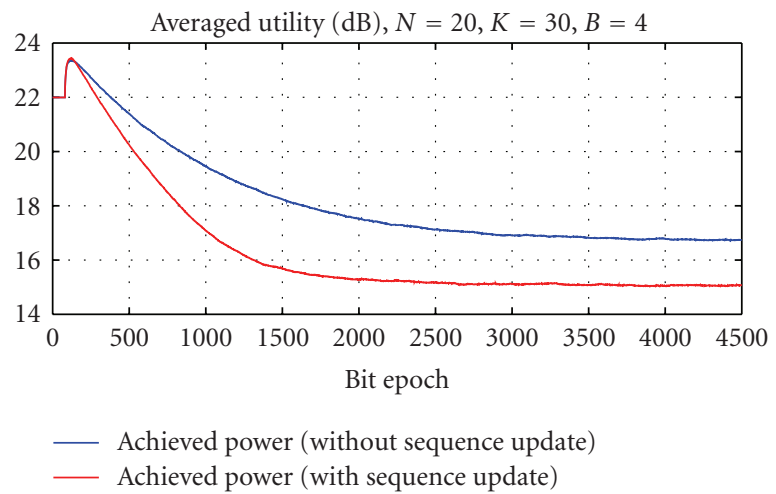

(b)

FIGURE 10: Multicell environment. Achieved utility and average transmit power (with and without spreading code optimization) versus time. Even though a formal proof of convergence is not available, it is seen that running some spreading code update iterations is beneficial to system performance.

capable of approaching the NE points of the nonadaptive versions of the games with good-to-excellent approximation.

The extension of the results of this paper to the case of asynchronous networks operating on multipath channels, a deeper investigation of the resource allocation problem in the multicell scenario, and a more comprehensive theoretical convergence analysis of the considered adaptive algorithms, are interesting and challenging research issues for future work in this area.

\section{Acknowledgments}

This paper was partly presented at the 2007 Tyrrhenian International Workshop on Digital Communications, Ischia Island, Italy, September 2007. This research was supported in part by the US National Science Foundation under Grants ANI-03-38807 and CNS-06-25637.

\section{References}

[1] G. Dimic, N. D. Sidiropoulos, and R. Zhang, "Medium access control-physical cross-layer design," IEEE Signal Processing Magazine, vol. 21, no. 5, pp. 40-50, 2004.

[2] C. Comaniciu, N. B. Mandayam, and H. V. Poor, Wireless Networks: Multiuser Detection in Cross-Layer Design, Springer, New York, NY, USA, 2005.

[3] D. Fudenberg and J. Tirole, Game Theory, MIT Press, Cambridge, Mass, USA, 1991.

[4] A. B. MacKenzie and S. B. Wicker, "Game theory in communications: motivation, explanation, and application to power control," in Proceedings of IEEE Global Telecommunications Conference (GLOBECOM '01), vol. 2, pp. 821-826, San Antonio, Tex, USA, November 2001.

[5] R. D. Yates, "A framework for uplink power control in cellular radio systems," IEEE Journal on Selected Areas in Communications, vol. 13, no. 7, pp. 1341-1347, 1995. 
[6] D. J. Goodman and N. B. Mandayam, "Power control for wireless data," IEEE Personal Communications, vol. 7, no. 2, pp. 48-54, 2000.

[7] C. U. Saraydar, N. B. Mandayam, and D. J. Goodman, "Efficient power control via pricing in wireless data networks," IEEE Transactions on Communications, vol. 50, no. 2, pp. 291303,2002

[8] C. U. Saraydar, Pricing and power control in wireless data networks, Ph.D. dissertation, Department of Electrical and Computer Engineering, Rutgers University, Piscataway, NJ, USA, 2001.

[9] F. Meshkati, H. V. Poor, S. C. Schwartz, and N. B. Mandayam, "An energy-efficient approach to power control and receiver design in wireless data networks," IEEE Transactions on Communications, vol. 53, no. 11, pp. 1885-1894, 2005.

[10] S. Buzzi and H. V. Poor, "Joint receiver and transmitter optimization for energy-efficient CDMA communications," IEEE Journal on Selected Areas in Communications, vol. 26, no. 3, pp. 459-472, 2008.

[11] S. Buzzi, V. Massaro, and H. V. Poor, "Power control and receiver design for energy efficiency in multipath CDMA channels with bandlimited waveforms," in Proceedings of the 41st Annual Conference on Information Sciences and Systems (CISS '07), pp. 282-287, John Hopkins University, Baltimore, Md, USA, March 2007.

[12] C. W. Sung, K. W. Shum, and K.-K. Leung, "Stability of distributed power and signature sequence control for CDMA systems-a game-theoretic framework," IEEE Transactions on Information Theory, vol. 52, no. 4, pp. 1775-1780, 2006.

[13] S. Haykin, Adaptive Filter Theory, Prentice Hall, Englewood Cliffs, NJ, USA, 3rd edition, 1996.

[14] M. Honig, U. Madhow, and S. Verdú, "Blind adaptive multiuser detection," IEEE Transactions on Information Theory, vol. 41, no. 4, pp. 944-960, 1995.

[15] J. Luo, S. Ulukus, and A. Ephremides, "Standard and quasistandard stochastic power control algorithms," IEEE Transactions on Information Theory, vol. 51, no. 7, pp. 2612-2624, 2005.

[16] S. Ulukus and A. Yener, "Iterative transmitter and receiver optimization for CDMA networks," IEEE Transactions on Wireless Communications, vol. 3, no. 6, pp. 1879-1884, 2004.

[17] G. S. Rajappan and M. L. Honig, "Signature sequence adaptation for DS-CDMA with multipath," IEEE Journal on Selected Areas in Communications, vol. 20, no. 2, pp. 384-395, 2002.

[18] P. Anigstein and V. Anantharam, "Ensuring convergence of the MMSE iteration for interference avoidance to the global optimum," IEEE Transactions on Information Theory, vol. 49, no. 4, pp. 873-885, 2003.

[19] S. Ulukus and R. D. Yates, "Iterative construction of optimum signature sequence sets in synchronous CDMA systems," IEEE Transactions on Information Theory, vol. 47, no. 5, pp. 19891998, 2001.

[20] S. M. Kay, Fundamentals of Statistical Signal Processing: Estimation Theory, Prentice Hall, Upper Saddle River, NJ, USA, 1993.

[21] C. U. Saraydar, N. B. Mandayam, and D. J. Goodman, "Pricing and power control in a multicell wireless data network," IEEE Journal on Selected Areas in Communications, vol. 19, no. 10, pp. 1883-1892, 2001. 